\title{
High Prevalence of Acute Exacerbation of Interstitial Lung Disease in Japanese Patients with Systemic Sclerosis
}

\author{
Fumiko Tomiyama, ${ }^{1}$ Ryu Watanabe, ${ }^{1}$ Tomonori Ishii, ${ }^{1}$ Yukiko Kamogawa, ${ }^{1}$ \\ Yoko Fujita, ${ }^{1}$ Yuko Shirota, ${ }^{1}$ Koichiro Sugimura, ${ }^{2}$ Hiroshi Fujiii ${ }^{1}$ and \\ Hideo Harigae ${ }^{1}$ \\ ${ }^{1}$ Department of Hematology and Rheumatology, Tohoku University Graduate School of Medicine, Sendai, Miyagi, \\ Japan \\ ${ }^{2}$ Department of Cardiovascular Medicine, Tohoku University Graduate School of Medicine, Sendai, Miyagi, Japan
}

\begin{abstract}
Systemic sclerosis (SSc) is a systemic autoimmune disease characterized by extensive fibrosis and autoantibodies. Its clinical manifestations are diverse and include Raynaud's phenomenon, gastrointestinal dysmotility, interstitial lung disease (ILD), pulmonary hypertension, and renal crisis. Among these, ILD is the primary cause of SSc-related death. It has been considered that acute exacerbation of ILD (AE-ILD) is not common in patients with SSc; however, little is known about the prevalence of AE-ILD in Japanese patients with SSc. In this study, we aimed to clarify the prevalence, clinical characteristics, and prognosis of patients with SSc who developed AE-ILD and to identify predictive factors for AE-ILD in our Japanese cohorts. Clinical data of patients who visited our department from 1990 to 2014 and fulfilled the 2013 classification criteria for SSc were retrospectively reviewed. A total of 139 patients were enrolled. The mean age of onset was 49.1 years, and 113 (81.3\%) patients were female; $116(83.5 \%)$ had limited cutaneous involvement, and the overall 10 -year survival rate was $92.0 \%$. Among $66(47.5 \%)$ patients with ILD, $13(9.4 \%)$ developed AE-ILD. Patients with AE-ILD had a significantly higher incidence of overlap with polymyositis (PM) or dermatomyositis (DM) and lower prevalence of anticentromere antibodies with higher mortality rate compared with those without AE-ILD. Multivariate Cox regression analysis identified that an overlap with PM or DM was the most significant predictive factor for AE-ILD. Our study results suggest that Japanese patients with SSc, particularly patients overlapped with PM or DM, have a high risk of AE-ILD.
\end{abstract}

Keywords: acute exacerbation; interstitial lung disease; overlap; polymyositis/dermatomyositis; systemic sclerosis Tohoku J. Exp. Med., 2016 August, 239 (4), 297-305. (C) 2016 Tohoku University Medical Press

\section{Introduction}

Systemic sclerosis (SSc) is a complex autoimmune disorder characterized by extensive fibrosis, vascular alterations, and autoantibodies (Gabrielli et al. 2009; Broen et al. 2014). SSc can affect almost any internal organ, and its clinical manifestations include Raynaud's phenomenon, gastrointestinal dysmotility, interstitial lung disease (ILD), pulmonary hypertension, and renal crisis. SSc is generally divided into two disease subsets based on the extent of skin sclerosis (LeRoy et al. 1988). One is limited type, in which skin thickening is limited to the areas distal to the elbows. The other is diffuse type, in which skin sclerosis extends proximal to the elbows (LeRoy et al. 1988). Patients with diffuse type have a high incidence of ILD, renal crisis, and gastrointestinal involvement; in contrast, patients with limited type frequently develop pulmonary hypertension (Gabrielli et al. 2009; Broen et al. 2014).
A variety of autoantibodies have been identified in patients with SSc (Nihtyanova and Denton 2010). Antitopoisomerase I antibody is associated with diffuse type and ILD, whereas anticentromere antibody is frequently detected in patients with limited type and pulmonary hypertension. Patients with positive anti-RNA polymerase III antibody have an increased risk of renal crisis and malignancies (Joseph et al. 2014; Moinzadeh et al. 2014). In addition to these antibodies, the anti-U1 ribonucleoprotein (RNP) antibody is occasionally found in patients who have features of other connective tissue disease named overlap syndrome (Kaji et al. 2014; Moinzadeh et al. 2015).

The causes of death in patients with SSc have dramatically changed over the past 30 years (Ioannidis et al. 2005; Steen and Medsger 2007). ILD, which occurs at a frequency of $34 \%-60 \%$ in patients with SSc (Meyer et al. 2007; Hunzelmann et al. 2008; Hashimoto et al. 2012), is currently the primary cause of SSc-related death (Ioannidis

Received April 9, 2016; revised and accepted July 15, 2016. Published online August 3, 2016; doi: 10.1620/tjem.239.297.

Correspondence: Ryu Watanabe, M.D., Ph.D., Department of Hematology and Rheumatology, Tohoku University Graduate School of Medicine, 1-1 Seiryo-machi, Aoba-ku, Sendai, Miyagi 980-8574, Japan.

e-mail: doctorwatanaberyu@yahoo.co.jp 
et al. 2005; Steen and Medsger 2007). However, no agents have been proven to effectively control ILD (Wells and Denton 2014). Cyclophosphamide was demonstrated to stabilize lung function in a randomized controlled trial, but its beneficial effect disappeared at 24 months (Tashkin et al. 2006; Tashkin et al. 2007). Moreover, acute exacerbation of ILD (AE-ILD) occurs at a 1-year frequency of $1.25 \%$ $3.3 \%$ and at a lifetime incidence of $7.2 \%$, with a high mortality rate in patients with connective tissue disease (Park et al. 2007; Suda et al. 2009). Given the chronic nature of the disease, it has been considered that AE-ILD is not common in patients with SSc (Fan et al. 2014; Cappelli et al. 2015), but recent papers suggest that AE-ILD may occur even in patients with SSc (Park et al. 2007; Bussone and Mouthon 2011; Tachikawa et al. 2012; Ando et al. 2013; Yasuoka 2016). However, little data are available regarding the prevalence of AE-ILD in Japanese patients with SSc.

Therefore, the aims of this study were to clarify the prevalence, clinical characteristics, treatment, and prognosis of Japanese patients with SSc who developed AE-ILD and to identify predictive factors for AE-ILD.

\section{Materials and Methods}

Study design

In this study, we enrolled 139 Japanese patients with SSc who visited our department from April 1990 to June 2014. All patients fulfilled the 2013 classification criteria (van den Hoogen et al. 2013). The follow-up period was defined as the time from the initial manifestation of SSc-related symptoms to either the date of death or the latest visit to our hospital. All patients were followed up until January 2015. The study protocol was approved by the ethics committees of Tohoku University Graduate School of Medicine and performed in accordance with the Declaration of Helsinki.

\section{Clinical data}

We retrospectively reviewed medical records and obtained data regarding the age of onset, gender, autoantibodies, physical findings, organ involvement, overlap of other connective tissue disease, history of malignancies, medications, and prognosis. The maximum values of Krebs von den Lungen-6 (KL-6) during the follow-up were also obtained. Limited or diffuse type was defined by the area of skin sclerosis, as previously described (LeRoy et al. 1988).

\section{Autoantibodies}

Anti-topoisomerase I antibody, anticentromere antibody, and anti-U1-RNP antibody were examined in all patients. Anti-RNA polymerase III antibody was tested only when patients had negative results for those autoantibodies.

\section{Evaluation of ILD}

The presence and progression of ILD were evaluated by radiologists using high-resolution computed tomography (HRCT). Follow-up CT scans were performed when patients experienced a worsening of respiratory symptoms or the serum KL-6 levels increased. We divided progression of ILD into three categories; no progression, slow progression, and acute exacerbation. The definition of acute exacerbation or AE-ILD included a prior diagnosis of ILD, worsening of dyspnea within 1 month, new ground-glass opacities on
HRCT, hypoxemia, and the exclusion of other causes for the worsening symptoms (Collard et al. 2007). If repeated CT scan showed progression without fulfilling the definition of AE-ILD, we defined it as slow progression.

\section{Other organ involvement}

With regard to reflux esophagitis, we included patients who received proton pump inhibitors for treating symptoms related to gastrointestinal dysmotility. Pulmonary hypertension was defined as a mean pulmonary artery pressure of $\geq 25 \mathrm{mmHg}$, which was confirmed by right-heart catheterization. Scleroderma renal crisis was diagnosed with renal biopsy (Nihtyanova et al. 2014).

Diagnosis of other connective tissue disease

The diagnoses of rheumatoid arthritis and Sjögren's syndrome were based on the 2010 criteria (Aletaha et al. 2010) and the 1999 Japanese Ministry of Health and Welfare's diagnostic criteria (Fujibayashi et al. 2004), respectively. The criteria of Bohan and Peter (Bohan and Peter 1975) were used to diagnose polymyositis (PM) and dermatomyositis (DM). With regard to the diagnosis of PM and DM, we included definite diagnosis alone.

\section{Treatment of ILD}

If patients with SSc had progressive ILD, we initially administered a moderate dose (up to $0.6 \mathrm{mg} / \mathrm{kg} / \mathrm{day}$ ) of oral prednisolone (PSL). When the progression of ILD was evident despite the administration of oral PSL, we intensified immunosuppressive therapy, including high-dose (up to $1 \mathrm{mg} / \mathrm{kg} / \mathrm{day}$ ) oral PSL, methylprednisolone pulse therapy, intravenous cyclophosphamide (IVCY), calcineurin inhibitor $(\mathrm{CI})$, and rituximab sometimes in combination.

\section{Statistical analysis}

Statistical analysis was performed using Graph Pad Prism 5 (San Diego, CA, USA) and EZR software (Kanda 2013), as previously described (Ishizuka et al. 2016; Yoshida et al. 2016). The Fisher's exact test was used for binary data, and the Mann-Whitney $U$-test was used for continuous data. Using the Kaplan-Meier method, the survival curves were drawn for a maximum of 10 years for each patient, and the log-rank test was used to compare the survival rates between patient groups. Stratified Cox regression analysis with a forward stepwise variable selection method was used to analyze any predictive factors. Variables with $\mathrm{p}$ values less than 1.0 in a univariate analysis were applied to the multivariate analysis. P values less than 0.05 were considered to be statistically significant.

\section{Results}

Demographic and baseline characteristics of patients with SSc

A total of 139 patients were enrolled. Baseline characteristics of the patients are shown in Table 1. The mean age of onset was $49.1 \pm 15.1$ years, and $81.3 \%$ of those patients were female. Anticentromere antibody was the most commonly identified autoantibody. Most of the patients (86.3\%) experienced Raynaud's phenomenon, whereas ILD was observed in 66 patients (47.5\%). 
Table 1. Baseline characteristics of enrolled patients with systemic sclerosis.

\begin{tabular}{|c|c|c|c|c|c|}
\hline \multicolumn{2}{|l|}{ Variables } & $\begin{array}{c}\text { All patients } \\
\mathrm{n}=139\end{array}$ & $\begin{array}{c}\text { Limited type } \\
\mathrm{n}=116\end{array}$ & $\begin{array}{c}\text { Diffuse type } \\
n=23\end{array}$ & $P$ value \\
\hline \multicolumn{2}{|c|}{ Age of onset, year (Mean \pm SD) } & $49.1 \pm 15.1$ & $50.6 \pm 13.9$ & $41.1 \pm 18.1$ & 0.027 \\
\hline \multicolumn{2}{|l|}{ Female } & $113(81.3)$ & $97(83.6)$ & $16(69.6)$ & 0.14 \\
\hline \multirow[t]{3}{*}{ Autoantibodies } & Anti-topoisomerase I & $37(26.6)$ & $27(23.3)$ & $10(43.5)$ & 0.068 \\
\hline & Anticentromere & $63(45.3)$ & $59(50.9)$ & $4(17.4)$ & 0.0051 \\
\hline & Anti-U1-RNP & $23(16.8)$ & $16(13.8)$ & $7(30.4)$ & 0.065 \\
\hline \multirow[t]{7}{*}{ Organ involvements } & Raynaud's phenomenon & $120(86.3)$ & $101(87.1)$ & $19(82.6)$ & 0.52 \\
\hline & Digital ulcer & $34(24.5)$ & $22(20.8)$ & $12(52.2)$ & 0.0036 \\
\hline & Nail fold bleeding & $57(41.0)$ & $45(38.8)$ & $12(52.2)$ & 0.25 \\
\hline & Reflux esophagitis & $68(48.9)$ & $54(44.6)$ & $18(78.3)$ & 0.0061 \\
\hline & Interstitial lung disease & $66(47.5)$ & $48(41.4)$ & $18(78.3)$ & 0.0013 \\
\hline & Pulmonary hypertension & $9(6.5)$ & $7(6.0)$ & $2(8.7)$ & 0.64 \\
\hline & Scleroderma renal crisis & $5(3.6)$ & $3(2.8)$ & $2(8.7)$ & 0.19 \\
\hline \multicolumn{2}{|l|}{ Malignancy } & $21(15.1)$ & $18(15.5)$ & $3(13.0)$ & 1.0 \\
\hline
\end{tabular}

$\mathrm{n},(\%)$. P values were determined by comparison between limited and diffuse type.

a

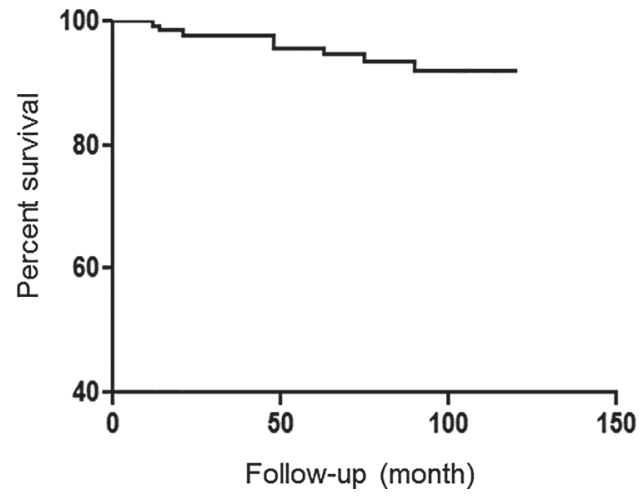

b

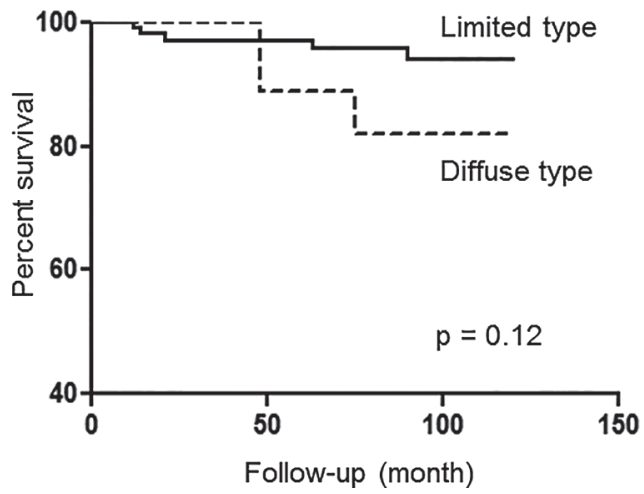

Fig. 1. Survival curve of patients with systemic sclerosis (SSc).

(a) Overall survival curve of 139 patients with SSc. (b) Survival curves of patients with limited $(\mathrm{n}=116)$ and diffuse (n =23) type.

Comparison of clinical characteristics between patients with limited and diffuse type

Of the 139 patients, $116(83.5 \%)$ were diagnosed with limited type. When we compared the clinical features between the two groups, patients with limited type had a significantly higher age of onset, a higher rate of anticentromere antibody, and a lower incidence of digital ulcers, reflux esophagitis, and ILD (Table 1).

\section{Overlap of other connective tissue disease}

A total of $28(20 \%)$ patients had an overlap with Sjögren's syndrome, $15(10 \%)$ with rheumatoid arthritis, and $8(5.8 \%)$ with PM or DM (hereafter we refer to as PM/ $\mathrm{DM})$. There were no significant differences in the prevalence of these coexisting diseases between patients with limited and diffuse type.

\section{Survival rates}

The overall survival rates for 139 patients were $99.2 \%$, $95.7 \%$, and $92.0 \%$ at 1,5 , and 10 years, respectively (Fig. 1a). The survival rate in patients with diffuse type tended to be lower than that in patients with limited type $(\mathrm{p}=0.12$, Fig. 1b).

\section{Radiological patterns of ILD}

Although CT scans were not available for two patients, the most common radiological finding of ILD was non-specific interstitial pneumonia, followed by usual interstitial pneumonia and organizing pneumonia (Fig. 2a).

\section{Clinical characteristics of patients who developed ILD}

Next, we compared clinical characteristics between patients with or without ILD (Table 2). Patients with ILD had a significantly higher KL-6 values and higher rates of anti-topoisomerase I antibody and renal crisis. The survival 
a

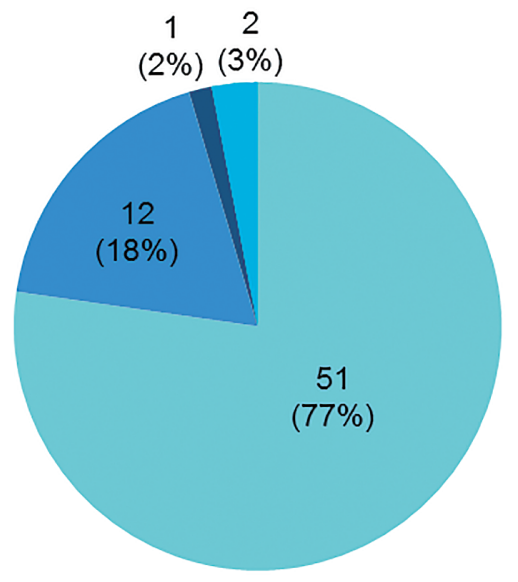

$\square$ Non-specific interstitial pneumonia

- Usual interstitial pneumonia

- Organizing pneumonia

Unknown b

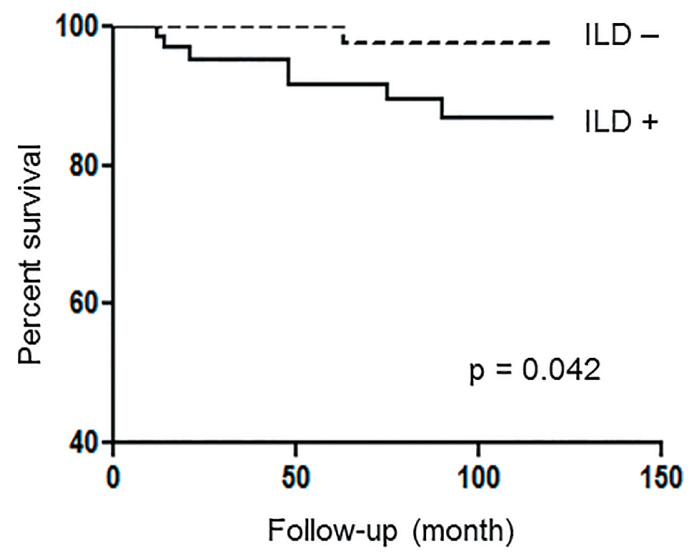

Fig. 2. Radiological findings of interstitial lung disease (ILD) and survival curves of patients with or without ILD.

(a) Radiological patterns of ILD observed in 66 patients. (b) Survival curves of patients with $(n=66)$ or without ILD (n $=73$ ).

Table 2. Comparison between patients with or without interstitial lung disease (ILD).

\begin{tabular}{|c|c|c|c|c|}
\hline \multicolumn{2}{|l|}{ Variables } & $\begin{array}{l}\text { ILD + } \\
\mathrm{n}=66\end{array}$ & $\begin{array}{l}\text { ILD - } \\
\mathrm{n}=73\end{array}$ & $P$ value \\
\hline \multicolumn{2}{|c|}{ Age of onset, year (Mean \pm SD) } & $49.2 \pm 14.8$ & $48.9 \pm 15.3$ & 0.94 \\
\hline \multicolumn{2}{|l|}{ Female } & $51(82.3)$ & $62(84.9)$ & 0.82 \\
\hline \multicolumn{2}{|l|}{ Limited type } & $48(72.7)$ & $68(93.2)$ & 0.0013 \\
\hline \multicolumn{2}{|c|}{ KL-6 (U/mL) (Median, range) } & $1,342(201-7,974)$ & $267(100-1,260)$ & $<0.0001$ \\
\hline \multirow[t]{3}{*}{ Autoantibodies } & Anti-topoisomerase I & $30(45.5)$ & $7(9.6)$ & $<0.0001$ \\
\hline & Anticentromere & $10(15.2)$ & $53(72.6)$ & $<0.0001$ \\
\hline & Anti-U1-RNP & $11(16.7)$ & $12(16.4)$ & 1.0 \\
\hline \multirow[t]{6}{*}{ Organ involvements } & Raynaud's phenomenon & $55(83.3)$ & $65(89.0)$ & 0.46 \\
\hline & Digital ulcer & $20(30.3)$ & $14(19.2)$ & 0.17 \\
\hline & Nail fold bleeding & $25(44.6)$ & $32(43.8)$ & 1.0 \\
\hline & Reflux esophagitis & $34(51.5)$ & $34(46.6)$ & 0.61 \\
\hline & Pulmonary hypertension & $6(9.1)$ & $3(4.1)$ & 0.31 \\
\hline & Scleroderma renal crisis & $5(7.6)$ & $0(0.0)$ & 0.022 \\
\hline \multicolumn{2}{|l|}{ Malignancy } & $13(19.7)$ & $8(11.0)$ & 0.16 \\
\hline
\end{tabular}

$\mathrm{n},(\%)$.

rate in patients with ILD was significantly lower than that in patients without ILD (Fig. 2b).

\section{Progression of ILD}

Among the 66 patients who developed ILD, 55 patients $(83 \%)$ underwent repeated CT scans. Of these patients, 22 patients showed no progression after a mean duration of 55 months (Fig. 3a). However, 33 patients demonstrated radiological progression of ILD after an aver- age duration of 23 months. Among them, 13 patients fulfilled the definition of AE-ILD. Thus, the prevalence of AE-ILD was $9.4 \%$ (13/139).

Clinical characteristics of patients who developed AE-ILD

Then, we compared clinical features between patients with AE-ILD and those without AE-ILD (Table 3). The patients with AE-ILD had significantly higher values of KL-6, a lower rate of anticentromere antibody, and greater 
a

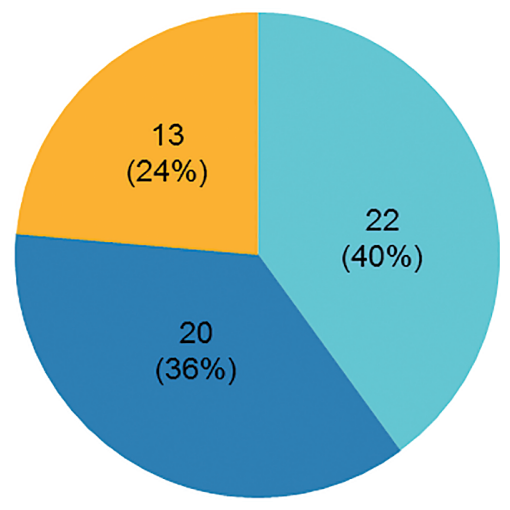

$\square$ No progression

Slow progression

Acute exacerbation b

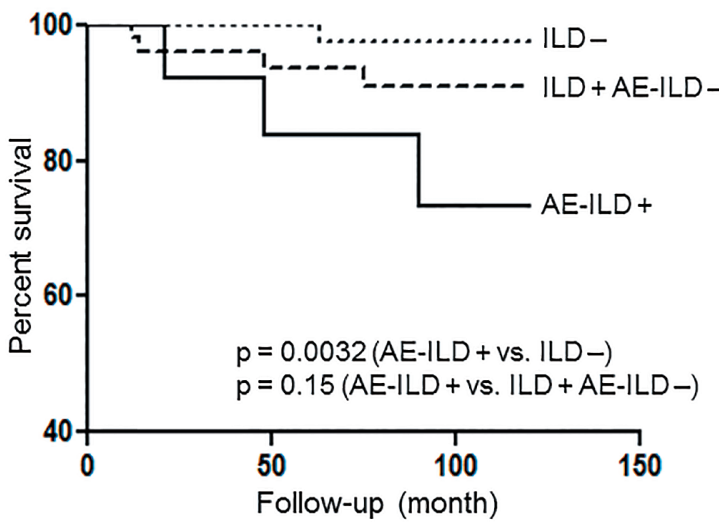

Fig. 3. Frequency of progression of interstitial lung disease (ILD) and comparison of survival rates between the groups sorted by ILD and acute exacerbation.

(a) Frequency of ILD progression among 55 patients who underwent repeated CT scans. The data from 11 out of 66 patients with ILD were not available. (b) Survival curves of patients who experienced acute exacerbation of ILD (AE-ILD+, $\mathrm{n}=13$ ), patients who had non- or slowly-progressive ILD (ILD+ AE-ILD-, $\mathrm{n}=53$ ), and patients who did not develop ILD (ILD-, $\mathrm{n}=73$ ).

Table 3. Comparison between patients with or without acute exacerbation of interstitial lung disease.

\begin{tabular}{|c|c|c|c|c|}
\hline \multicolumn{2}{|l|}{ Variables } & $\begin{array}{c}\text { Acute } \\
\text { exacerbation }+ \\
n=13\end{array}$ & $\begin{array}{c}\text { Acute } \\
\text { exacerbation - } \\
n=126\end{array}$ & P value \\
\hline \multicolumn{2}{|c|}{ Age of onset, year (Mean \pm SD) } & $49.3 \pm 12.7$ & $49.0 \pm 15.3$ & 0.87 \\
\hline \multicolumn{2}{|l|}{ Female } & $10(76.9)$ & $103(81.7)$ & 0.71 \\
\hline \multicolumn{2}{|l|}{ Limited type } & $9(69.2)$ & $107(84.9)$ & 0.23 \\
\hline \multicolumn{2}{|c|}{ KL-6 (U/mL) (Median, range) } & $2,313(870-5,784)$ & $403(100-7,974)$ & $<0.0001$ \\
\hline \multirow[t]{3}{*}{ Autoantibodies } & Anti-topoisomerase I & $4(30.8)$ & $33(26.2)$ & 0.75 \\
\hline & Anticentromere & $2(15.4)$ & $61(48.4)$ & 0.037 \\
\hline & Anti-U1-RNP & $3(23.1)$ & $20(15.9)$ & 0.45 \\
\hline \multirow[t]{6}{*}{ Organ involvements } & Raynaud's phenomenon & $11(84.6)$ & $109(86.5)$ & 0.69 \\
\hline & Digital ulcer & $3(23.1)$ & $31(24.6)$ & 1.0 \\
\hline & Nail fold bleeding & $6(46.2)$ & $51(40.5)$ & 0.77 \\
\hline & Reflux esophagitis & $5(38.5)$ & $63(50.0)$ & 0.56 \\
\hline & Pulmonary hypertension & $2(15.4)$ & $7(5.6)$ & 0.20 \\
\hline & Scleroderma renal crisis & $0(0.0)$ & $5(4.1)$ & 1.0 \\
\hline Overlap & Polymyositis/dermatomyositis & $3(23.1)$ & $5(4.0)$ & 0.028 \\
\hline Malignancy & & $1(7.7)$ & $20(15.9)$ & 0.69 \\
\hline
\end{tabular}

$\mathrm{n},(\%)$.

incidence of overlap with PM/DM than the patients without AE-ILD. There was no significant difference in the prevalence of overlap with Sjögren's syndrome and rheumatoid arthritis between the two groups. Fig. $3 b$ showed the survival curves of three groups: patients without ILD, patients with AE-ILD, and patients without AE-ILD. The survival rate of patients with AE-ILD was significantly lower than that of patients without ILD $(p=0.0032)$. A decreased trend was observed in patients with AE-ILD, compared to those without AE-ILD $(p=0.15)$.

\section{Treatment of ILD}

Among the 66 patients with ILD, 49 (74\%) patients received immunosuppressive therapy (Fig. 4a). Of these, 20 patients received oral PSL alone, whereas the rest of the patients received other immunosuppressive agents. When 
a Interstitial lung disease + $\mathrm{n}=66$

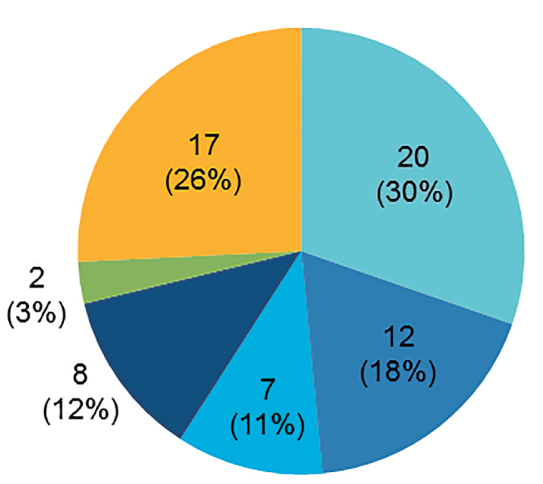

PSL alone
PSL +Cl
PSL+IVCY
PSL+Cl+IVCY
Cl alone
No immunosuppressive agents b Acute exacerbation +

$n=13$

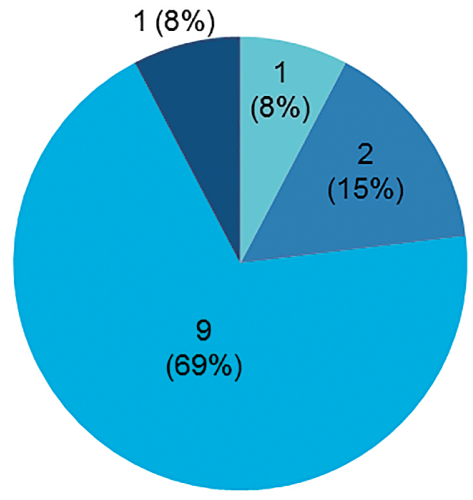

- PSLalone

$\square \mathrm{PSL}+\mathrm{Cl}$

- PSL +IVCY

- $\mathrm{PSL}+\mathrm{Cl}+\mathrm{IVCY}$

\section{Cl; calcineurin inhibitor, IVCY; intravenous cyclophosphamide, PSL; prednisolone}

Fig. 4. Treatment of patients with interstitial lung disease (ILD).

(a) Overall treatment of patients with ILD $(n=66)$. (b) Treatment for patients who developed acute exacerbation of ILD $(\mathrm{n}=13)$.

CI, calcineurin inhibitor; IVCY, intravenous cyclophosphamide; PSL, prednisolone.

Table 4. Multivariate Cox regression analysis for acute exacerbation of interstitial lung disease.

\begin{tabular}{|c|c|c|c|c|c|}
\hline \multirow[b]{2}{*}{ Variables } & \multirow[b]{2}{*}{ Adverse factor } & \multicolumn{2}{|c|}{ Univariate } & \multicolumn{2}{|c|}{ Multivariate } \\
\hline & & $\mathrm{HR}(95 \% \mathrm{CI})$ & $\mathrm{P}$ value & $\mathrm{HR}(95 \% \mathrm{CI})$ & P value \\
\hline Age of onset & +1 & $\begin{array}{c}10.24 \\
(0.982-1.068) \\
1.559\end{array}$ & 0.27 & & \\
\hline Gender & male & $\begin{array}{c}(0.4284-5.676) \\
2.005\end{array}$ & 0.50 & & \\
\hline Limited / diffuse type & diffuse type & $\begin{array}{c}(0.6126-6.559) \\
1.124\end{array}$ & 0.25 & & \\
\hline Anti-topoisomerase I & positive & $\begin{array}{c}(0.345-3.662) \\
0.2721\end{array}$ & 0.85 & 0.3065 & \\
\hline Anticentromere & positive & $\begin{array}{c}(0.06011-1.231) \\
1.339\end{array}$ & 0.09 & $(0.0673-1.396)$ & 0.13 \\
\hline Anti-U1-RNP & positive & $\begin{array}{c}(0.3639-4.927) \\
0.8641\end{array}$ & 0.66 & & \\
\hline Raynaud's phenomenon & positive & $\begin{array}{c}(0.191-3.908) \\
0.6955\end{array}$ & 0.85 & & \\
\hline Reflux esophagitis & positive & $\begin{array}{c}(0.232-2.085) \\
0.9305\end{array}$ & 0.52 & & \\
\hline Digital ulcer & positive & $\begin{array}{c}(0.2558-3.384) \\
2.122\end{array}$ & 0.91 & & \\
\hline Pulmonary hypertension & positive & $\begin{array}{c}(0.4672-9.638) \\
0.386\end{array}$ & 0.33 & & \\
\hline Malignancy & positive & $\begin{array}{c}(0.05012-2.973) \\
1.534\end{array}$ & 0.36 & & \\
\hline Overlap with Sjögren's syndrome & positive & $\begin{array}{c}(0.4704-5.001) \\
1.152\end{array}$ & 0.48 & & \\
\hline Overlap with rheumatoid arthritis & positive & $\begin{array}{c}(0.254-5.222) \\
8.084\end{array}$ & 0.85 & 6.973 & \\
\hline Overlap with polymyositis/dermatomyositis & positive & $(2.107-31.01)$ & 0.0023 & $(1.8050-26.930)$ & 0.0049 \\
\hline
\end{tabular}

CI, confidence interval; HR, hazard ratio. 
limited to the patients who developed AE-ILD (Fig. 4b), most of the patients received combination therapy. However, 6 out of 13 patients (46\%) died during follow-up.

\section{Multivariate analysis of predictive factors for AE-ILD}

We performed multivariate Cox regression analysis to clarify the predictive factors for AE-ILD (Table 4). The age of onset, gender, disease subsets, autoantibodies, organ involvement, malignancies, and overlap with connective tissue disease were employed in this analysis. Univariate analysis suggested that anticentromere antibody and the overlap with $\mathrm{PM} / \mathrm{DM}$ were significant variables; however, multivariate analysis showed that the most important predictive factor was the overlap with PM/DM $(\mathrm{p}=0.0049)$.

\section{Discussion}

In this study, we retrospectively analyzed the clinical characteristics of 139 Japanese SSc patients and demonstrated that the prevalence of AE-ILD was $9.4 \%$ and that coexisting $\mathrm{PM} / \mathrm{DM}$ was the most significant predictive factor. To our knowledge, this is the first study that showed the prevalence, clinical characteristics, outcome, and predictive factor of AE-ILD in Japanese patients with SSc.

Clinical characteristics of our patient cohort were consistent with previous reports (Gabrielli et al. 2009; Broen et al. 2014; Nihtyanova et al. 2014), since patients with limited type had higher prevalence of anticentromere antibody and a lower frequency of internal organ involvement compared with patients with diffuse type (Table 1 and Fig. 1b). However, the ratio of limited type to diffuse type (116/23, 5.0) was higher than that in previous studies (Hashimoto et al. 2011; Nihtyanova et al. 2014; Shirai et al. 2015). For example, the ratio was $1.7(252 / 146)$ at the Royal Free Hospital in the UK (Nihtyanova et al. 2014), 2.1 (273/132) at Kitasato University in Japan (Hashimoto et al. 2011), and $2.9(127 / 44)$ at Keio University in Japan (Shirai et al. 2015). In addition, the 10-year survival rate in our study $(92.0 \%)$ was greater than that found in previous studies from Japan and Western countries (72.6\%-88.0\%) (Jacobsen et al. 2001; Czirjak et al. 2008; Hashimoto et al. 2011). The reason for these discrepancies remains unclear; however, the prevalence of limited type without internal organ involvement has been increasing in recent years (Ferri et al. 2014), which may have attributed to these differences.

In our study, the prevalence of ILD was $47.5 \%$ and the most common HRCT finding of ILD was non-specific interstitial pneumonia (Fig. 2a). Patients with ILD had a significantly higher rate of anti-topoisomerase I antibody and a decreased survival rate compared with patients without ILD (Table 2 and Fig. 2b). These findings were also consistent with previous reports (Hashimoto et al. 2011; Wells and Denton 2014). Moreover, AE-ILD occurred at a frequency of $9.4 \%(13 / 139)$ in our study. Previous papers showed that the 1 -year frequency of acute exacerbation was $1.25 \%$ $3.3 \%$ and a lifetime incidence $7.2 \%$ in patients with connective tissue disease (Park et al. 2007; Suda et al. 2009).
When limited to Japanese patients with SSc, there are three case series concerning AE-ILD (Suda et al. 2009; Tachikawa et al. 2012; Ando et al. 2013). Suda et al. (2009) reported that none out of 13 patients showed AE-ILD during median follow-up of 6 years; however, Ando et al. (2013) showed that 3 out of 71 patients $(4.2 \%)$ died of AE-ILD during 9.8 years of follow-up. Our study result showing that 6 out of 139 patients (4.3\%) died of AE-ILD is consistent with the latter (Ando et al. 2013). The 1-year frequency of AE-ILD in patients with idiopathic pulmonary fibrosis (IPF) was reportedly 5\%-15\% (Ryerson et al. 2015). We cannot directly compare our SSc cohort and IPF cohort; however, our study suggests that the prevalence of AE-ILD in Japanese patients with SSc is not low.

The clinical characteristics of patients who developed AE-ILD showed a greater incidence of overlap with PM/ $\mathrm{DM}$, and multivariate analysis indicated that an overlap with $\mathrm{PM} / \mathrm{DM}$ was the most significant predictive factor (Tables 3 and 4). Suda et al. (2009) suggested that advanced age was a risk factor for acute exacerbation in connective tissue disease-related ILD, but the age of onset was not an associated factor in our study (Table 4). About $10 \%-20 \%$ of patients with SSc had overlapping features with other rheumatic diseases (Kowal-Bielecka et al. 2009; Pakozdi et al. 2011). Pakozdi et al. (2011) reported that the most common connective tissue disease was PM/DM, followed by rheumatoid arthritis and Sjögren's syndrome. It is still controversial whether SSc overlap syndrome is a separate disease entity or should be included in the two disease subsets of limited and diffuse type. However, a recent study has clearly demonstrated that its clinical course was different from that of limited or diffuse type with respect to autoantibodies and disease progression (Moinzadeh et al. 2015). The prevalence of AE-ILD in SSc overlap syndrome was not mentioned in this previous study (Moinzadeh et al. 2015), but our study suggests that Japanese patients with SSc-myositis overlap syndrome have a high risk of AE-ILD.

With regard to the treatment for AE-ILD, there is no consensus concerning appropriate management (Mathai and Danoff 2016). Therefore, we treated AE-ILD patients by combination therapy with a high dose of PSL and other immunosuppressive agents, similar to the treatment of patients who developed acute exacerbation of IPF (Kondoh et al. 1993; Azuma et al. 2005). However, the mortality rate was extremely high $(6 / 13,46 \%)$, and thus a more appropriate therapeutic strategy is required.

This study has also several limitations: 1) this is a retrospective, observational study. Therefore, a large prospective cohort study is essential to confirm our findings; 2 ) our data did not include the modified Rodnan total skin thickness score, which is the most common technique for the measurement of skin involvement (Furst et al. 1998; Clements et al. 2000); 3) anti-RNA polymerase III antibody was examined only when patients had negative results for other autoantibodies; and 4) autoantibodies that can be 
detected in patients with SSc overlap syndrome (e.g., antiPM-Scl antibody, anti-Ku antibody, and anti-RuvBL1/2) could not be examined at our institute (Kaji et al. 2014). 5) Our conclusion would simply reflect that PM/DM-related ILD has a high risk of AE. But even if we exclude patients with SSc-myositis overlap syndrome, 10 out of 131 patients (7.6\%) developed AE-ILD, which again means that AE-ILD is not rare in SSc patients.

In conclusion, our study results demonstrated that 9.4\% of SSc patients developed AE-ILD during the disease course and that coexisting PM/DM was the most significant predictive factor for acute exacerbation. The prognosis of patients who developed AE-ILD remains extremely poor, therefore a novel therapeutic strategy is urgently required.

\section{Conflict of Interest}

The authors declare no conflict of interest.

\section{References}

Aletaha, D., Neogi, T., Silman, A.J., Funovits, J., Felson, D.T., Bingham, C.O. 3rd., Birnbaum, N.S., Burmester, G.R., Bykerk, V.P., Cohen, M.D., Combe, B., Costenbader, K.H., Dougados, M., Emery, P., Ferraccioli, G., et al. (2010) 2010 Rheumatoid arthritis classification criteria: an American College of Rheumatology/European League Against Rheumatism collaborative initiative. Arthritis Rheum., 62, 2569-2581.

Ando, K., Motojima, S., Doi, T., Nagaoka, T., Kaneko, N., Aoshima, M. \& Takahashi, K. (2013) Effect of glucocorticoid monotherapy on pulmonary function and survival in Japanese patients with scleroderma-related interstitial lung disease. Respir. Investig., 51, 69-75.

Azuma, A., Nukiwa, T., Tsuboi, E., Suga, M., Abe, S., Nakata, K., Taguchi, Y., Nagai, S., Itoh, H., Ohi, M., Sato, A. \& Kudoh, S. (2005) Double-blind, placebo-controlled trial of pirfenidone in patients with idiopathic pulmonary fibrosis. Am. J. Respir. Crit. Care Med., 171, 1040-1047.

Bohan, A. \& Peter, J.B. (1975) Polymyositis and dermatomyositis (first of two parts). N. Engl. J. Med., 292, 344-347.

Broen, J.C., Radstake, T.R. \& Rossato, M. (2014) The role of genetics and epigenetics in the pathogenesis of systemic sclerosis. Nat. Rev. Rheumatol., 10, 671-681.

Bussone, G. \& Mouthon, L. (2011) Interstitial lung disease in systemic sclerosis. Autoimmun. Rev., 10, 248-255.

Cappelli, S., Bellando-Randone, S., Camiciottoli, G., De Paulis, A., Guiducci, S. \& Matucci-Cerinic, M. (2015) Interstitial lung disease in systemic sclerosis: where do we stand? Eur. Respir. Rev., 24, 411-419.

Clements, P.J., Hurwitz, E.L., Wong, W.K., Seibold, J.R., Mayes, M., White, B., Wigley, F., Weisman, M., Barr, W., Moreland, L., Medsger, T.A. Jr., Steen, V.D., Martin, R.W., Collier, D., Weinstein, A., et al. (2000) Skin thickness score as a predictor and correlate of outcome in systemic sclerosis: high-dose versus low-dose penicillamine trial. Arthritis Rheum., 43, 2445-2454.

Collard, H.R., Moore, B.B., Flaherty, K.R., Brown, K.K., Kaner, R.J., King, T.E. Jr., Lasky, J.A., Loyd, J.E., Noth, I., Olman, M.A., Raghu, G., Roman, J., Ryu, J.H., Zisman, D.A., Hunninghake, G.W., et al. (2007) Acute exacerbations of idiopathic pulmonary fibrosis. Am. J. Respir. Crit. Care Med., 176, 636-643.

Czirjak, L., Kumanovics, G., Varju, C., Nagy, Z., Pakozdi, A., Szekanecz, Z. \& Szucs, G. (2008) Survival and causes of death in 366 Hungarian patients with systemic sclerosis. Ann. Rheum. Dis., 67, 59-63.
Fan, M.H., Feghali-Bostwick, C.A. \& Silver, R.M. (2014) Update on scleroderma-associated interstitial lung disease. Curr. Opin. Rheumatol., 26, 630-636.

Ferri, C., Sebastiani, M., Lo Monaco, A., Iudici, M., Giuggioli, D., Furini, F., Manfredi, A., Cuomo, G., Spinella, A., Colaci, M., Govoni, M. \& Valentini, G. (2014) Systemic sclerosis evolution of disease pathomorphosis and survival. Our experience on Italian patients' population and review of the literature. Autoimmun. Rev., 13, 1026-1034.

Fujibayashi, T., Sugai, S., Miyasaka, N., Hayashi, Y. \& Tsubota, K. (2004) Revised Japanese criteria for Sjögren's syndrome (1999): availability and validity. Mod. Rheumatol., 14, 425-434.

Furst, D.E., Clements, P.J., Steen, V.D., Medsger, T.A. Jr., Masi, A.T., D'Angelo, W.A., Lachenbruch, P.A., Grau, R.G. \& Seibold, J.R. (1998) The modified Rodnan skin score is an accurate reflection of skin biopsy thickness in systemic sclerosis. J. Rheumatol., 25, 84-88.

Gabrielli, A., Avvedimento, E.V. \& Krieg, T. (2009) Scleroderma. N. Engl. J. Med., 360, 1989-2003.

Hashimoto, A., Endo, H., Kondo, H. \& Hirohata, S. (2012) Clinical features of 405 Japanese patients with systemic sclerosis. Mod. Rheumatol., 22, 272-279.

Hashimoto, A., Tejima, S., Tono, T., Suzuki, M., Tanaka, S., Matsui, T., Tohma, S., Endo, H. \& Hirohata, S. (2011) Predictors of survival and causes of death in Japanese patients with systemic sclerosis. J. Rheumatol., 38, 1931-1939.

Hunzelmann, N., Genth, E., Krieg, T., Lehmacher, W., Melchers, I., Meurer, M., Moinzadeh, P., Muller-Ladner, U., Pfeiffer, C., Riemekasten, G., Schulze-Lohoff, E., Sunderkoetter, C., Weber, M., Worm, M., Klaus, P., et al. (2008) The registry of the German Network for Systemic Scleroderma: frequency of disease subsets and patterns of organ involvement. Rheumatology (Oxford), 47, 1185-1192.

Ioannidis, J.P., Vlachoyiannopoulos, P.G., Haidich, A.B., Medsger, T.A. Jr., Lucas, M., Michet, C.J., Kuwana, M., Yasuoka, H., van den Hoogen, F., Te Boome, L., van Laar, J.M., Verbeet, N.L., Matucci-Cerinic, M., Georgountzos, A. \& Moutsopoulos, H.M. (2005) Mortality in systemic sclerosis: an international meta-analysis of individual patient data. Am. J. Med., 118, $2-10$.

Ishizuka, M., Watanabe, R., Ishii, T., Machiyama, T., Akita, K., Fujita, Y., Shirota, Y., Fujii, H. \& Harigae, H. (2016) Longterm follow-up of 124 patients with polymyositis and dermatomyositis: Statistical analysis of prognostic factors. Mod. Rheumatol., 26, 115-120.

Jacobsen, S., Ullman, S., Shen, G.Q., Wiik, A. \& Halberg, P. (2001) Influence of clinical features, serum antinuclear antibodies, and lung function on survival of patients with systemic sclerosis. J. Rheumatol., 28, 2454-2459.

Joseph, C.G., Darrah, E., Shah, A.A., Skora, A.D., Casciola-Rosen, L.A., Wigley, F.M., Boin, F., Fava, A., Thoburn, C., Kinde, I., Jiao, Y., Papadopoulous, N., Kinzler, K.W., Volgelstein, B. \& Rosen, A. (2014) Association of the autoimmune disease scleroderma with an immunologic response to cancer. Science, 343, 152-157.

Kaji, K., Fertig, N., Medsger, T.A. Jr., Satoh, T., Hoshino, K., Hamaguchi, Y., Hasegawa, M., Lucas, M., Schnure, A., Ogawa, F., Sato, S., Takehara, K., Fujimoto, M. \& Kuwana, M. (2014) Autoantibodies to RubBL1 and Rub BL2: a novel systemic sclerosis-related antibody associated with diffuse cutaneous and skeletal muscle involvement. Arthritis Care Res., 66, 575-584.

Kanda, Y. (2013) Investigation of the freely available easy-to-use software 'EZR' for medical statistics. Bone Marrow Transplant., 48, 452-458.

Kondoh, Y., Taniguchi, H., Kawabata, Y., Yokoi, T., Suzuki, K. \& Takagi, K. (1993) Acute exacerbation in idiopathic pulmonary fibrosis. Analysis of clinical and pathological findings in three 
cases. Chest, 103, 1808-1812.

Kowal-Bielecka, O., Landewe, R., Avouac, J., Chwiesko, S., Miniati, I., Czirjak, L., Clements, P., Denton, C., Farge, D., Fligelstone, K., Foldvari, I., Furst, D.E., Muller-Ladner, U., Seibold, J., Silver, R.M., et al. (2009) EULAR recommendations for the treatment of systemic sclerosis: a report from the EULAR Scleroderma Trials and Research group (EUSTAR). Ann. Rheum. Dis., 68, 620-628.

LeRoy, E.C., Black, C., Fleischmajer, R., Jablonska, S., Krieg, T., Medsger, T.A. Jr., Rowell, N. \& Wollheim, F. (1988) Scleroderma (systemic sclerosis): classification, subsets and pathogenesis. J. Rheumatol., 15, 202-205.

Mathai, S.C. \& Danoff, S.K. (2016) Management of interstitial lung disease associated with connective tissue disease. $B M J$, 352, h6819.

Meyer, O.C., Fertig, N., Lucas, M., Somogyi, N. \& Medsger, T.A. Jr. (2007) Disease subsets, antinuclear antibody profile, and clinical features in 127 French and 247 US adult patients with systemic sclerosis. J. Rheumatol., 34, 104-109.

Moinzadeh, P., Aberer, E., Ahmadi-Simab, K., Blank, N., Distler, J.H., Fierlbeck, G., Genth, E., Guenther, C., Hein, R., Henes, J., Herich, L., Herrgott, I., Koetter, I., Kreuter, A., Krieg, T., et al. (2015) Disease progression in systemic sclerosis-overlap syndrome is significantly different from limited and diffuse cutaneous systemic sclerosis. Ann. Rheum. Dis., 74, 730-737.

Moinzadeh, P., Fonseca, C., Hellmich, M., Shah, A.A., Chighizola, C., Denton, C.P. \& Ong, V.H. (2014) Association of anti-RNA polymerase III autoantibodies and cancer in scleroderma. Arthritis Res. Ther., 16, R53.

Nihtyanova, S.I. \& Denton, C.P. (2010) Autoantibodies as predictive tools in systemic sclerosis. Nat. Rev. Rheumatol., 6, 112-116.

Nihtyanova, S.I., Schreiber, B.E., Ong, V.H., Rosenberg, D., Moinzadeh, P., Coghlan, J.G., Wells, A.U. \& Denton, C.P. (2014) Prediction of pulmonary complications and long-term survival in systemic sclerosis. Arthritis Rheum., 66, 16251635 .

Pakozdi, A., Nihtyanova, S., Moinzadeh, P., Ong, V.H., Black, C.M. \& Denton, C.P. (2011) Clinical and serological hallmarks of systemic sclerosis overlap syndromes. J. Rheumatol., 38, 2406-2409.

Park, I.N., Kim, D.S., Shim, T.S., Lim, C.M., Lee, S.D., Koh, Y., Kim, W.S., Kim, W.D., Jang, S.J. \& Colby, T.V. (2007) Acute exacerbation of interstitial pneumonia other than idiopathic pulmonary fibrosis. Chest, 132, 214-220.

Ryerson, C.J., Cottin, V., Brown, K.K. \& Collard, H.R. (2015) Acute exacerbation of idiopathic pulmonary fibrosis: shifting the paradigm. Eur. Repir. J., 46, 512-520.
Shirai, Y., Okazaki, Y., Inoue, Y., Tamura, Y., Yasuoka, H., Takeuchi, T. \& Kuwana, M. (2015) Elevated levels of pentraxin 3 in systemic sclerosis: associations with vascular manifestations and defective vasculogenesis. Arthritis Rheumatol., 67, 498-507.

Steen, V.D. \& Medsger, T.A. (2007) Changes in causes of death in systemic sclerosis, 1972-2002. Ann. Rheum. Dis., 66, 940-944.

Suda, T., Kaida, Y., Nakamura, Y., Enomoto, N., Fujisawa, T., Imokawa, S., Hashizume, H., Naito, T., Hashimoto, D., Takehara, Y., Inui, N., Nakamura, H., Colby, T.V. \& Chida, K. (2009) Acute exacerbation of interstitial pneumonia associated with collagen vascular diseases. Respir. Med., 103, 846-853.

Tachikawa, R., Tomii, K., Ueda, H., Nagata, K., Nanjo, S., Sakurai, A., Otsuka, K., Kaji, R., Hayashi, M., Katakami, N. \& Imai, Y. (2012) Clinical features and outcome of acute exacerbation of interstitial pneumonia: collagen vascular disease-related versus idiopathic. Respiration, 83, 20-27.

Tashkin, D.P., Elashoff, R., Clements, P.J., Goldin, J., Roth, M.D., Furst, D.E., Arriola, E., Silver, R., Strange, C., Bolster, M., Seibold, J.R., Riley, D.J., Hsu, V.M., Varga, J., Schraufnagel, D.E., et al. (2006) Cyclophosphamide versus placebo in scleroderma lung disease. N. Engl. J. Med., 354, 2655-2666.

Tashkin, D.P., Elashoff, R., Clements, P.J., Roth, M.D., Furst, D.E., Silver, R.M., Goldin, J., Arriola, E., Strange, C., Bolster, M., Seibold, J.R., Riley, D.J., Hsu, V.M., Varga, J., Schraufnagel, D.E., et al. (2007) Effects of 1-year treatment with cyclophosphamide on outcomes at 2 years in scleroderma lung disease. Am. J. Respir. Crit. Care Med., 176, 1026-1034.

van den Hoogen, F., Khanna, D., Fransen, J., Johnson, S.R., Baron, M., Tyndall, A., Matucci-Cerinic, M., Naden, R.P., Medsger, T.A. Jr., Carreira, P.E., Riemekasten, G., Clements, P.J., Denton, C.P., Distler, O., Allnore, Y., et al. (2013) 2013 classification criteria for systemic sclerosis: an American College of Rheumatology/European League against Rheumatism collaborative initiative. Arthritis Rheum., 65, 2737-2747.

Wells, A.U. \& Denton, C.P. (2014) Interstitial lung disease in connective tissue disease-mechanisms and management. Nat. Rev. Rheumatol., 10, 728-739.

Yasuoka, H. (2016) Recent treatments of interstitial lung disease with systemic sclerosis. Clin. Med. Insights Circ. Respir. Pulm. Med., 9, 97-110.

Yoshida, M., Watanabe, R., Ishii, T., Machiyama, T., Akita, K., Fujita, Y., Shirota, Y., Sugimura, K., Fujii, H., Shimokawa, H. \& Harigae, H. (2016) Retrospective analysis of 95 patients with large vessel vasculitis: a single center experience. Int. J. Rheum. Dis., 19, 87-94. 